Supplement of Biogeosciences, 12, 5075-5092, 2015

http://www.biogeosciences.net/12/5075/2015/

doi:10.5194/bg-12-5075-2015-supplement

(C) Author(s) 2015. CC Attribution 3.0 License.

(c) (i)

Supplement of

\title{
Effects of fluctuating hypoxia on benthic oxygen consumption in the Black Sea (Crimean shelf)
}

\section{A. Lichtschlag et al.}

Correspondence to: A. Lichtschlag (alic@noc.ac.uk)

The copyright of individual parts of the supplement might differ from the CC-BY 3.0 licence. 


\section{Supplement Figures and Tables}
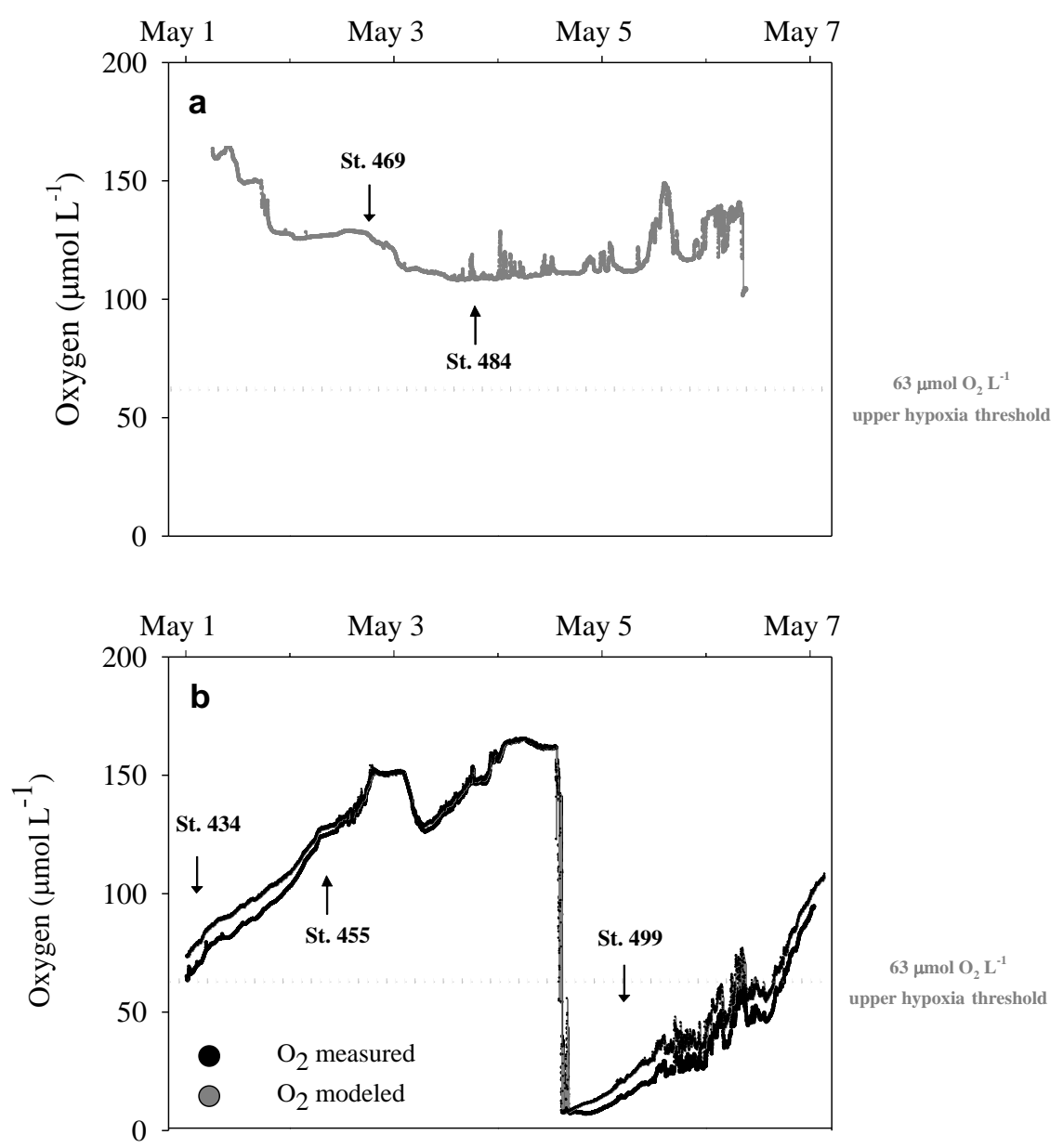

Fig. S1: Stationary moorings with sensors measuring one meter above the sediment over a time period of 7 days; a) the oxygen concentration from the mooring at $100 \mathrm{~m}$ was modeled from recorded density data; bottom water was always oxic during the measurements, still strong variations (up to $60 \mu \mathrm{mol} \mathrm{O}_{2} \mathrm{~L}^{-1}$ ) were visible during the deployment time; b) at the mooring at $135 \mathrm{~m}$ water depth, measuring $1.5 \mathrm{~m}$ above the sediment, the water-column oxygen concentration strongly varied between oxic and hypoxic conditions, dropping to nearly anoxic conditions on May $5^{\text {th }}$. Time points where oxygen consumption was measured at these two water depths are indicated. The horizontal line indicates the conventional hypoxia threshold concentration of 63 $\mu \mathrm{mol} \mathrm{O} \mathrm{L}^{-1}$. 

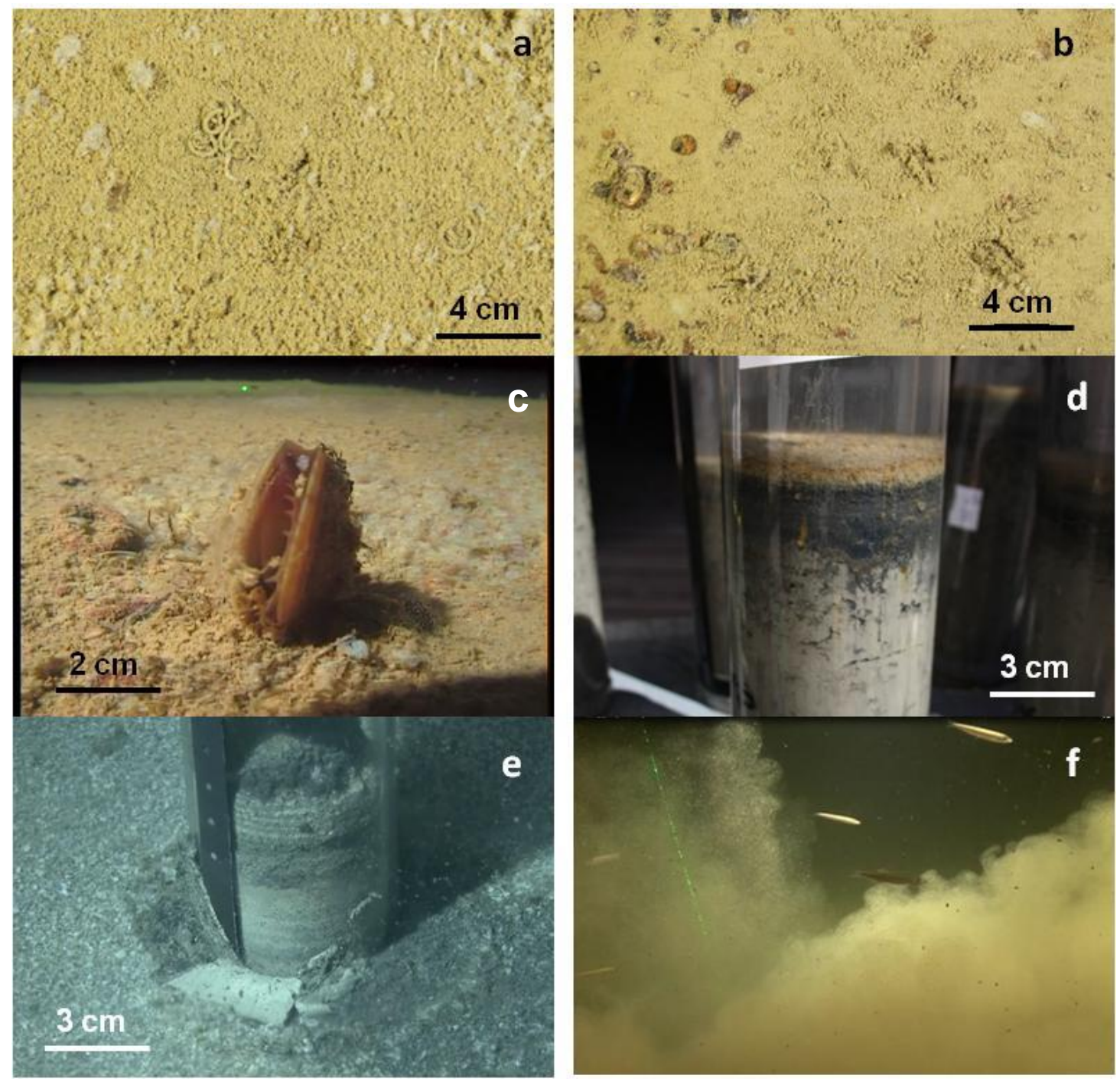

Fig. S2: Images of seafloor and sediments; a) fecal structures on top of the sediment in the oxic zone, b) brown iron-encrusted shells in the oxic zone, c) living bivalve on top of the sediment in the oxic zone; d) vertical layering of the sediment with oxygenated sediment on top in the oxic zone; e) vertical layering of the sediment

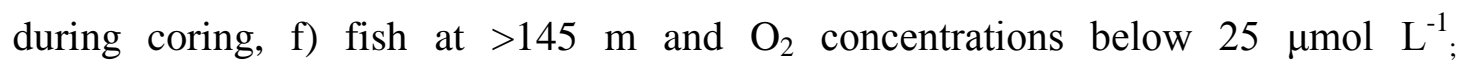
photographs are copyright JAGO-Team GEOMAR. 


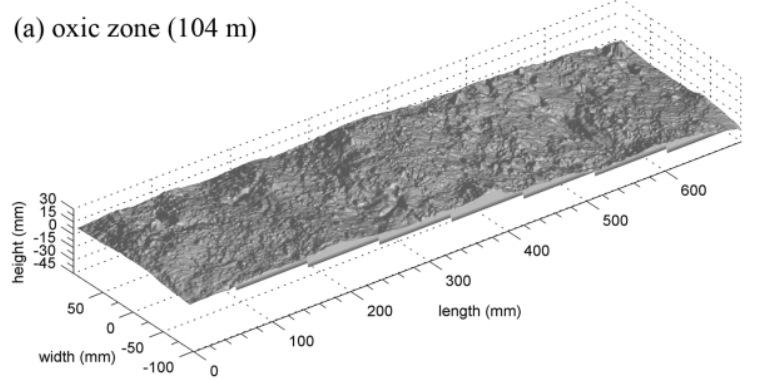

(c) hypoxic-anoxic zone (155 m)
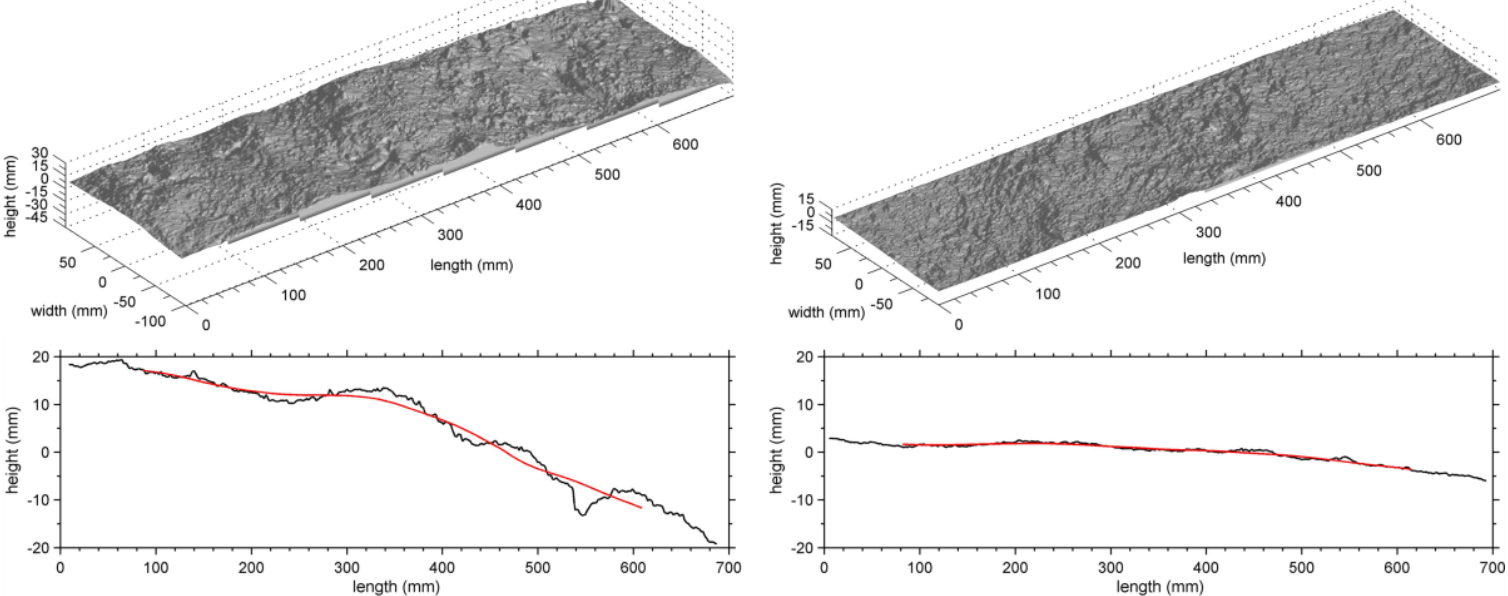

(b) oxic-hypoxic zone $(138 \mathrm{~m})$

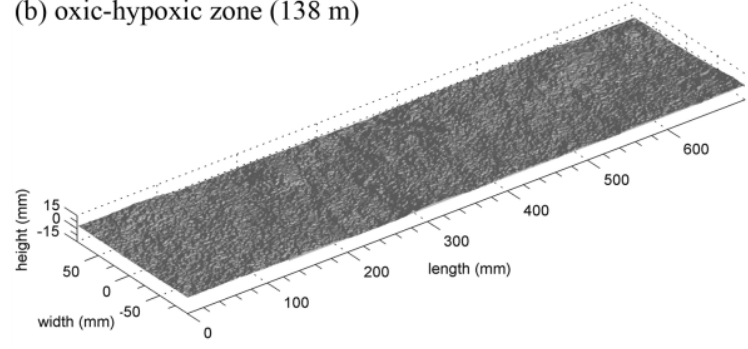

(d) anoxic-sulfidic zone (206 m)
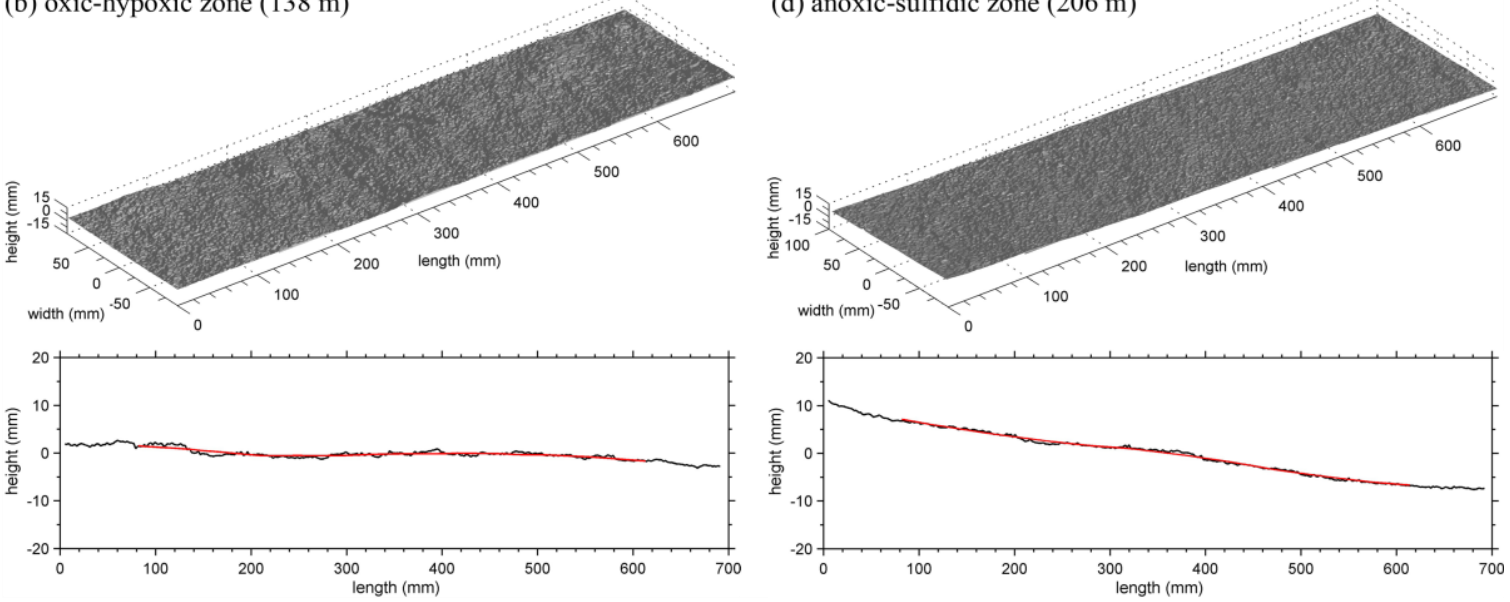

Fig. S3: Shaded 3D surfaces showing examples of micro-topography measurements obtained at (a) 104, (b) 138, (c) 155, and (d) $206 \mathrm{~m}$ water depth. The 2D plots show topography profiles extracted along the center line of the respective surfaces (black line). The red line shows the running average of the same profile $(155 \mathrm{~mm}$ averaging window). Deviations of the profile from profiles smoothed at different window sizes were used to compare roughness between stations (see section 2.3 and 3.5) 

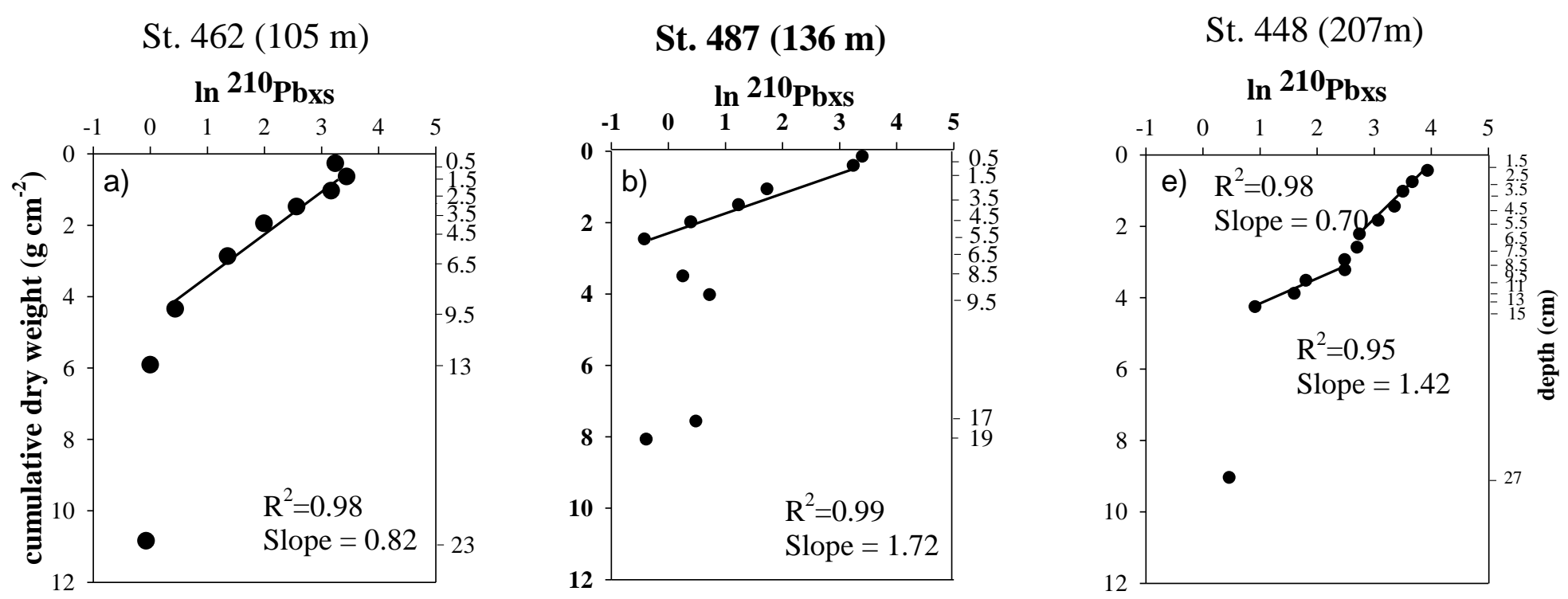

Fig. S4: Profiles of excess ${ }^{210} \mathrm{~Pb}$ activity, cumulative dry weight (left y-axis) and depth (right y-axis). Regression lines are plotted for data that were included in calculations of sediment accumulation rates. 
Table S1: Macrofauna composition and abundance (x $10^{3}$ individuals $\mathrm{m}^{-2}$ ) per sampling depth (m) at the outer Western Crimean Shelf (not quantitative for the entire size class). Results were grouped according to bottom-water oxygenation zones and integrated over the upper $5 \mathrm{~cm}$.

\begin{tabular}{|c|c|c|c|c|c|c|c|c|c|c|c|c|c|}
\hline $\begin{array}{l}\text { Taxa } \\
\left(\times 10^{3} \text { individuals } \mathrm{m}^{-2}\right)\end{array}$ & 101 & 104 & 105 & 117 & 120 & 129 & 138 & 138 & 145 & 151 & 155 & 162 & 163 \\
\hline Ascidiacea & 0.2 & 0 & 0 & 0 & 0 & 0 & 0 & 0 & 0 & 0 & 0 & 0 & 0 \\
\hline Bivalvia & 0.1 & 0.4 & 0 & 0 & 0 & 0 & 0 & 0 & 0 & 0 & 0 & 0 & 0 \\
\hline Cnidaria & 2.1 & 0.4 & 1.4 & 7.5 & 50.1 & 53.1 & 35.4 & 23.4 & 1.7 & 0 & 1.4 & 1 & 0 \\
\hline Gastropoda & 0 & 0.4 & 0 & 0 & 0 & 0 & 0 & 0 & 0 & 0 & 0 & 0.2 & 0 \\
\hline Nemertini & 0 & 0 & 0.5 & 0 & 0 & 0 & 0 & 0 & 0 & 0 & 0 & 0 & 0 \\
\hline Oligochaeta & 0.8 & 0.3 & 3.1 & 1.6 & 0.6 & 0.7 & 14.3 & 2.6 & 0.7 & 0 & 0 & 0 & 0.1 \\
\hline Polychaeta & 1.6 & 0.8 & 1.7 & 0.7 & 0.1 & 0 & 1.7 & 0.3 & 0 & 0 & 0 & 0 & 0 \\
\hline Porifera & 0.2 & 0 & 0 & 0 & 0 & 0 & 0 & 0 & 0 & 0 & 0 & 0 & 0 \\
\hline$\Sigma$ & 5 & 1.9 & 6.7 & 9.8 & 50.8 & 53.8 & 51.4 & 26.3 & 2.4 & 0 & 1.4 & 1.2 & 0.1 \\
\hline Zone & & & & oxic & & & oxi & poxic & \multicolumn{5}{|c|}{ hypoxic-anoxic } \\
\hline
\end{tabular}


Table S2: Meiofauna composition and abundance (x10 ${ }^{4}$ individuals $\mathrm{m}^{-2}$ ) per sampling depth $(\mathrm{m})$ at the outer Western Crimean Shelf. Results were grouped according to bottom-water oxygenation zones and integrated over the upper $5 \mathrm{~cm}$.

\begin{tabular}{|c|c|c|c|c|c|c|c|c|c|c|c|c|c|}
\hline $\begin{array}{l}\text { Taxa } \\
\left(\mathrm{x10}^{4} \text { individuals } \mathrm{m}^{-2}\right)\end{array}$ & 101 & 104 & 105 & 117 & 120 & 129 & 138 & 138 & 145 & 151 & 155 & 162 & 163 \\
\hline Acari & 0 & 0.26 & 0 & 0 & 0.01 & 0.04 & 0.05 & 0 & 0 & 0 & 0 & 0 & 0 \\
\hline Amphipoda & 0 & 0 & 0 & 0.02 & 0 & 0 & 0 & 0 & 0 & 0 & 0 & 0 & 0 \\
\hline Bivalvia & 0.45 & 0.2 & 0.74 & 0.17 & 0.16 & 0.08 & 0.29 & 0.23 & 0.88 & 0 & 0.04 & 0.31 & 0.06 \\
\hline Ciliophora & 1.52 & 0.16 & 3.08 & 0.81 & 1.52 & 4.91 & 0.79 & 3.55 & 3.49 & 12.41 & 1.02 & 3.32 & 0.97 \\
\hline Cnidaria & 0.59 & 0.03 & 0.29 & 0.07 & 1.14 & 0.63 & 0.02 & 3.8 & 0.98 & 0.14 & 0 & 1.36 & 0.04 \\
\hline Forams hard shelled & 0.25 & 0.09 & 0.05 & 0.05 & 0.16 & 7.39 & 13.39 & 15.42 & 2.49 & 0.24 & 0.23 & 1.05 & 0.01 \\
\hline Forams soft shelled & 1.05 & 0.37 & 2.8 & 2.22 & 3.56 & 7.17 & 13.27 & 7.68 & 17.88 & 10.45 & 1.28 & 3.83 & 0.19 \\
\hline Gastropoda & 0.01 & 0.03 & 0 & 0.02 & 0.01 & 0.04 & 0 & 0.01 & 0 & 0 & 0.01 & 0 & 0 \\
\hline Gromia & 1.13 & 0.66 & 0.53 & 2.36 & 3.32 & 2.01 & 4.57 & 2.28 & 0.6 & 1.24 & 1.41 & 0.81 & 0 \\
\hline Harpacticoida & 1.7 & 1.3 & 3.99 & 0.66 & 0.76 & 1.45 & 7.91 & 3.46 & 0.14 & 0 & 0.27 & 0.02 & 0.19 \\
\hline Kinorhyncha & 0.41 & 0.12 & 0.62 & 0.11 & 0.32 & 0.05 & 0.17 & 0.01 & 0 & 0 & 0 & 0 & 0 \\
\hline Nauplia Decapoda & 0.93 & 0.26 & 0.12 & 0 & 0 & 0.02 & 0 & 0.43 & 0 & 0 & 0 & 0 & 0 \\
\hline Nematoda & 221.5 & 128.75 & 248.78 & 91.98 & 183.06 & 131.62 & 183.82 & 134.44 & 82.78 & 30.66 & 25.03 & 31.23 & 3.36 \\
\hline Nemertini & 0 & 0.01 & 0 & 0 & 0 & 0 & 0 & 0.01 & 0 & 0 & 0 & 0 & 0 \\
\hline Oligochaeta juvenile & 0.02 & 0.32 & 0 & 0 & 0.01 & 0.38 & 0.98 & 0.04 & 0 & 0 & 0 & 0 & 0 \\
\hline Ostracoda & 2.88 & 2.8 & 6.74 & 0.05 & 0 & 0 & 0.14 & 0.01 & 0 & 0 & 0 & 0 & 0 \\
\hline Polychaeta & 1.12 & 0.66 & 1.86 & 0.23 & 0.58 & 0.92 & 0.74 & 0.42 & 0.22 & 0.12 & 0.16 & 0.19 & 0.18 \\
\hline Tardigrada & 1.11 & 0.57 & 1.55 & 0 & 0 & 0 & 0 & 0 & 0 & 0 & 0 & 0 & 0 \\
\hline Turbellaria & 0.69 & 1.38 & 2.18 & 1.66 & 4.33 & 0.07 & 0.12 & 0.33 & 0.02 & 0.02 & 0.04 & 0 & 0 \\
\hline Others & 0.65 & 0 & 0.05 & 0 & 0.51 & 0 & 0 & 0.04 & 0.02 & 0 & 0.03 & 0 & 0 \\
\hline$\Sigma$ & 236.0 & 138.0 & 273.4 & 100.4 & 199.5 & 156.8 & 226.3 & 172.2 & 109.5 & 55.3 & 29.5 & 42.1 & 5.0 \\
\hline Zone & & & & oxic & & & \multicolumn{2}{|c|}{ oxic-hypoxic } & \multicolumn{5}{|c|}{ hypoxic-anoxic } \\
\hline
\end{tabular}


Table S3: Meiofauna community dissimilarity per sampling depths. Upper triangle: dissimilarity (based on Bray-Curtis), values closer to 1 represent high dissimilarity. Lower triangle: percentage of shared taxa. Colors depict oxygenation regimes, oxic (blue), oxic-hypoxic (pink), hypoxic-anoxic (red).

\begin{tabular}{|c|c|c|c|c|c|c|c|c|c|c|c|c|c|}
\hline & 101 & 104 & 105 & 117 & 120 & 129 & 138 & 138 & 145 & 151 & 155 & 162 & 163 \\
\hline 101 & & 0.1 & 0.1 & 0.2 & 0.2 & 0.2 & 0.3 & 0.3 & 0.3 & 0.4 & 0.2 & 0.3 & 0.4 \\
\hline 104 & 89 & & 0.1 & 0.2 & 0.2 & 0.3 & 0.3 & 0.3 & 0.4 & 0.5 & 0.3 & 0.4 & 0.4 \\
\hline 105 & 88 & 78 & & 0.2 & 0.2 & 0.3 & 0.3 & 0.3 & 0.3 & 0.4 & 0.2 & 0.3 & 0.3 \\
\hline 117 & 76 & 68 & 75 & & 0.1 & 0.2 & 0.2 & 0.2 & 0.3 & 0.3 & 0.2 & 0.3 & 0.3 \\
\hline 120 & 76 & 78 & 65 & 75 & & 0.2 & 0.2 & 0.2 & 0.3 & 0.3 & 0.2 & 0.3 & 0.3 \\
\hline 129 & 82 & 83 & 71 & 71 & 93 & & 0.1 & 0.1 & 0.2 & 0.3 & 0.1 & 0.2 & 0.3 \\
\hline 145 & 62 & 56 & 71 & 71 & 71 & 67 & 71 & 62 & & 0.2 & 0.2 & 0.1 & 0.3 \\
\hline 151 & $\mathbf{5 0}$ & 44 & 57 & 57 & 57 & 53 & 57 & 50 & 80 & & 0.3 & 0.2 & 0.3 \\
\hline 155 & 62 & 56 & 60 & 71 & 71 & 67 & 60 & 62 & 82 & 64 & & 0.2 & 0.3 \\
\hline 162 & 56 & 50 & 64 & 64 & 64 & 60 & 64 & 56 & 90 & 70 & 73 & & 0.2 \\
\hline
\end{tabular}

